\title{
Article
}

\section{Sensitivity and specificity of CTC for the detection of colonic neoplasia after positive fecal occult blood testing: Systematic review and meta-analysis}

\begin{abstract}
Objective: CT colonography (CTC) is recommended after positive fecal occult blood testing (FOBt) when colonoscopy is incomplete or infeasible. We aimed to estimate the sensitivity and specificity of CTC for colorectal cancer and adenomatous polyps following positive FOBt via systematic review.

Methods: The MEDLINE, EMBASE, AMED and Cochrane Library databases were searched for CTC studies reporting sensitivity and specificity for colorectal cancer and adenomatous polyps. Included subjects had tested FOBt-positive by guaiac or immunochemical methods. Per-patient detection rates were summarized via forest plots. Meta-analysis of sensitivity and specificity was conducted using a bivariate random effects model and the average operating point calculated.
\end{abstract}

Results: Of 538 articles considered, 5 met inclusion criteria, describing results from 622 patients. Research study quality was good. CTC had a high per-patient average sensitivity of $88.8 \%$ (95\% Cl 83.6 to $92.5 \%$ ) for $\geq 6 \mathrm{~mm}$ adenomas or colorectal cancer, with low betweenstudy heterogeneity. Specificity was both more heterogeneous and lower, at an average of $75.4 \%$ (95\%Cl 58.6 to $86.8 \%)$.

Conclusion: Few studies have investigated CTC in FOBt-positive individuals. CTC is sensitive at $a \geq 6 \mathrm{~mm}$ threshold but specificity is lower and variable. Despite the limited data, these results suggest CTC may adequately substitute for colonoscopy when the latter is undesirable.

Keywords CT colonography, Colorectal Neoplasms, Screening, Occult blood, Review, Systematic

\section{Key points}

- Faecal occult blood testing is the commonest mass-screening test for colorectal cancer.

- Few studies specifically evaluate CT colonography after positive faecal occult blood testing.

- $\quad$ CTC is approximately $89 \%$ sensitive for $\geq 6 \mathrm{~mm}$ adenomas/cancer in this setting.

- Specificity is lower, at approximately $75 \%$, and more variable.

- CT colonography is a good alternative when colonoscopy is undesirable.

\section{Abbreviations}

$\mathrm{CAD}=$ computer assisted detection

$\mathrm{CTC}=$ computed tomographic colonography

$\mathrm{CRC}=$ colorectal cancer

gFOBt = guaiac faecal occult blood test 
FIT = faecal immunochemical test

NPV $=$ negative predictive value

PPV $=$ positive predictive value

QUADAS-2 = quality assessment of diagnostic accuracy studies, second revision 


\section{Introduction}

Worldwide, over half a million people die from colorectal cancer (CRC)[1] annually. Population screening reduces mortality in two ways: Established malignancy is detected sooner, facilitating cure; and precursor polyps (namely adenomas) can be removed, preventing cancer development subsequently. Internationally, most screening programmes use faecal occult blood testing[2], which is proven in meta-analysis of randomised trials to reduce CRC-related mortality[3]. Faecal testing (whether by guaiac-based techniques, gFOBt, or immunochemical techniques, FIT) is widely available[4], acceptable to screenees[5] and cost-effective[6].

Merely demonstrating occult blood loss cannot improve outcomes unless cancer is found and treated, or large adenomas removed. For most, colonoscopy both confirms cancer and permits endoluminal excision of smaller adenomas and cancers. However, colonoscopy may be incomplete, contraindicated or refused by some screenees[7]. To maximize neoplasia detection, an alternative test is required. One possibility is CT colonography (CTC), which has been recommended when colonoscopy is not feasible or incomplete[8]. This recommendation is largely based on meta-analysis of cohort studies[9-11] and two randomised trials of symptomatic older patients $[12,13]$. Whilst extrapolation from such literature is intuitively logical, gFOBt/FIT-positive screenees have such a high prevalence of abnormality that subsequent tests require extremely high sensitivity to achieve an acceptable negative predictive value. Additionally, screen-detected cancers are earlier stage than symptomatic tumours[14], and could be more difficult to detect at CTC. Furthermore, advanced histologic features are more common in $\mathrm{gFOBt} / \mathrm{FIT}$-positives, even at equivalent adenoma diameter[15, 16]. Since CTC has less sensitivity for small polyps[10,17], this implies more advanced neoplasia will be missed when testing gFOBt/FIT-positive subjects than asymptomatic individuals, in whom subcentimetre adenomas rarely harbour advanced neoplasia[18].

If CTC is to be adopted widely following positive gFOBt/FIT, sensitivity and specificity for cancer and adenomas should be known with precision. The relevant comparator is colonoscopy, since it is accurate, widely available and generally safe[7]. Without this information, clinicians and patients are unable to balance the risks of colonoscopy against the chance of missing neoplasia with CTC. To address this, we performed a systematic review and meta-analysis of the sensitivity and specificity of CTC for colorectal cancer and adenomatous polyps in gFOBt/FIT-positive individuals.

\section{Materials and Methods}

\section{Data sources}

A literature search of the MEDLINE database was performed using Pubmed (http://www.ncbi.nlm.nih.gov/pubmed). All primary studies for the period January 1994 (the year CT colonography was first described) to February 2013 were considered. To retrieve articles relevant to stool testing, the Medical Subject Headings (MeSH) terms feces, Occult Blood and Immunologic Tests were combined with the free-text terms faeces, feces, faecal, fecal, FIT, iFOB ${ }^{*}, F O B^{*}$ and occult. This search was combined with a search for CTC-related literature using the MeSH terms colonography, colography, CT colonography, CT colonoscopy, CT pneumocolon, virtual colonoscopy or virtual endoscopy and the free text terms colonography, colography, CTC, computerized tomographic colonoscopy, computed tomographic colonoscopy and CT pneumocolon. Subsequently, the Cochrane library, EMBASE, AMED and OVID were searched using the free text terms $f e c^{*}$, face* $F O B^{*}$, gFOB ${ }^{*}$, iFOB ${ }^{*}, F I T$, immunochem ${ }^{*}$ and immunolog ${ }^{*}$ combined with CT comput tomogra*, colonogra*, virtua* colonosc ${ }^{*}$ and virtua* endosc ${ }^{*}$. Reference lists from reports eventually selected were also searched manually. 


\section{Study selection}

Studies were eligible if the patient population had tested positive for faecal occult blood and been imaged by CTC (defined below). Studies describing other populations were potentially eligible if separate per-patient data were presented for $\mathrm{gFOBt} / \mathrm{FIT}$-positive participants. Only full reports of original data from in vivo research in human subjects were considered. Review articles, editorials, commentaries, book chapters, abstracts, guidelines and position statements were ineligible.

\section{Target disorder}

To be included, the focus of the study had to be the detection of colorectal neoplasia using CTC in comparison with a reference test. Studies assessing a technical development (for example, computer-assisted detection, CAD) or alteration in CTC technique were potentially eligible if results were presented for CTC examinations conducted according to consensus standards[19].

\section{CT test methods}

On the basis of consensus documents for the performance of CTC[19, 20], all patients had to undergo bowel preparation (either cleansing, tagging or both) prior to imaging in a minimum of two positions, with or without intravenous contrast. Interpretation of CTC before the reference test (or blinding of the observer to reference test findings) was required. No stipulation was made regarding the mode of interpretation used by CTC observers, nor regarding the use of CAD.

\section{Reference test}

All CTC findings had to be verified by a reference test. Conventional colonoscopy, segmental unblinded colonoscopy and surgery (with subsequent histopathology) were acceptable alternatives.

\section{Data extraction}

All abstracts of primary studies were independently screened by two authors (AAP and DAP) who excluded clearly ineligible studies. The full text of potentially eligible studies was retrieved and scrutinised. Differences in opinion regarding eligibility were resolved in face-toface consensus. From each eligible study, the following were extracted; (a) publication year, (b) number and age range of gFOBt/FIT-positive subjects, (c) single- or multi-centre study design, (d) CTC technique (reconstruction interval, use of cathartics, stool tagging, intravenous contrast medium and the approximate radiation dose), (e) approximate experience of CTC readers, (f) interpretation strategy (including two-dimensional or threedimensional viewing, use of CAD, and double-reporting), (g) reference standard against which CTC and colonoscopy were compared, (h) number of patients with cancer by the reference standard, (i) per-patient sensitivity of CTC for cancer, (j) per-patient sensitivity of colonoscopy for cancer, (k) number of patients with $\geq 10 \mathrm{~mm}$ and $\geq 6 \mathrm{~mm}$ polyps and adenomas, including advanced adenomas, by the reference standard, (l) per-patient sensitivity of CTC for $\geq 10 \mathrm{~mm}$ and $\geq 6 \mathrm{~mm}$ polyps, adenomas and advanced adenomas, $(\mathrm{m})$ per-patient sensitivity of colonoscopy for $\geq 10 \mathrm{~mm}$ and $\geq 6 \mathrm{~mm}$ polyps, adenomas and advanced adenomas, ( $n$ ) specificity of CTC for polyps, adenomas and advanced adenomas (at $\geq 10 \mathrm{~mm}$ and $\geq 6 \mathrm{~mm}$ thresholds) and (o) positive and negative predictive values of CTC for cancers, polyps, adenomas and advanced adenomas at $\geq 6 \mathrm{~mm}$ and $\geq 10 \mathrm{~mm}$ thresholds.

Specificity of CTC for cancers cannot be calculated because large polyps and cancers are 
only distinguishable post-hoc (i.e. histologically). Article quality was judged using QUADAS-2 (quality assessment tool of diagnostic accuracy studies)[21].

\section{Analysis}

Numbers of included and excluded studies (and reasons for exclusion), patient characteristics, study design, CTC technique, observer experience and viewing mode were tabulated and analysed with descriptive statistics. The QUADAS-2 assessment was converted into a summary score of either "high risk", "low risk" or "unclear", for both the risk of bias and concern over applicability to the systematic review question, as recommended by the QUADAS-2 authors[21].

Per-patient 2x2 contingency tables were constructed for meta-analysis of sensitivity and specificity. Forest plots of sensitivity and specificity were generated using the forest command of the metafor package[22] for R version 2.15.1[23]. Heterogeneity between primary studies was assessed using the $\mathrm{I}^{2}$ statistic, with values of $25 \%, 50 \%$ and $75 \%$ taken to indicate low, moderate and high heterogeneity respectively. Meta-analysis of paired sensitivity and specificity was conducted via a bivariate random effects model that enables estimation of a summary receiver operating characteristic curve using the $\mathrm{R}$ package mada. The results for single-reader CTC were used, since this is the most frequent mode of interpretation in current clinical practice. Bivariate models allow for possible correlation between sensitivity and specificity[24, 25]. The following factors that might increase heterogeneity were considered as moderator covariates in the bivariate model: (a) year of publication; (b) number of included participants; (c) prevalence of $6-9 \mathrm{~mm}$ and $\geq 10 \mathrm{~mm}$ adenomas or carcinoma; (d) single- or multi-centre design; (e) use of faecal tagging; (f) reader experience; and $(\mathrm{g})$ use of three-dimensional interpretation. Covariates $(\mathrm{a}),(\mathrm{b})$ and (c) were treated as continuous variables, and (d) to $(\mathrm{g})$ as binary variables.

\section{Results \\ Search results}

A flow diagram of abstracts examined and articles retrieved, included and excluded (with reasons) is shown in Figure 1. In summary, 122 studies were identified from the Pubmed and Cochrane Library search and 416 from the EMBASE, AMED and OVID search. 39 fulltext articles were screened and ultimately 5 were included. Excluded studies are detailed in the Appendix.

\section{Characteristics of included studies}

Five articles were included, reporting four distinct studies[26-30]. Two of these articles reported different primary outcome measures for the same patient cohort[26, 27]. All four studies were performed in Europe: One in the Netherlands[26, 27], one in Italy[29], one in France[30] and one international study in Italy and Belgium[28]. The Italian study was from a single centre, the Netherlands study used two centres, the French study used 26 centres and the international study used 21 centres initially, although only 12 contributed patients to the final analysis. The two articles reporting the same patient group were both included (as relevant data were presented across the two articles) but individual subjects were not duplicated during analysis.

\section{Patient characteristics and CTC technique}

A total of $622 \mathrm{gFOBt} / \mathrm{FIT}$-positive patients were enrolled in the selected studies, ranging from 49 to 302 per study (Table 1). Two studies were designed specifically to assess gFOBt/FITpositive patients[26, 27, 29] whereas the other two included gFOBt-positive patients as a subgroup of other high-risk populations[28, 30]. Only the results of $\mathrm{gFOBt} / \mathrm{FIT}$-positive 
subjects are included here. The age range was 50 to 75 years (one study reported mean and interquartile range[30]). Prevalence of $\geq 6 \mathrm{~mm}$ adenomas or cancer ranged from 32.0 to $65.3 \%$. Cathartic bowel preparation was used by all except Liedenbaum et al, who used a reduced-laxative regime. Faecal tagging was used variably (see Table). All studies used dual patient positioning, multislice CT, low dose $(<100 \mathrm{mAs})$, unenhanced acquisition and narrow reconstruction intervals. Reading strategy was left to radiologist preference in two studies[28, 30] and primary 2D in the other two[26, 27, 29]. Computer-aided detection (CAD) was not used. Liedenbaum et al reported results for both single- and double-reporting[26]. A minimum level of radiologist experience was required by all studies, ranging from 50 to 100 cases. The reference standard was universally segmental unblinded colonoscopy (i.e. initial colonoscopy optimised by re-examination following revelation of CTC findings).

\section{Study quality}

Overall research study quality was good. In one study, 10 patients were excluded because CTC images were judged non-diagnostic and a further 2 had incomplete colonoscopy[26, 27]. In clinical practice, a variable proportion of patients will have poor quality CTC and it is not possible to simply exclude them. However, such cases were a small proportion of the total number in this particular study (12 exclusions, 302 participants), implying a negligible effect on overall results. In another report, patient flow through the study was not reported separately for gFOBt-positive participants[30]. All studies used segmental unblinded colonoscopy as the reference standard, a practice which theoretically may lead to incorporation bias. The summary QUADAS-2 results are presented in Table 2.

\section{Sensitivity and negative predictive value}

Two studies $[27,29]$ reported the sensitivity of CTC for colorectal cancer separately from the sensitivity for adenomas. Sensitivity for CRC was 100\% in one study[29] (2 of 2 cancers detected) and $95.5 \%$ in the other (21 of 22 cancers detected)[27]. The two studies describing CTC for high risk patients[28, 30] (including some gFOBt/FIT-positives) did not report sensitivity for cancer in the gFOBt/FIT-positive subset. Initial colonoscopy did not miss any cancers (vs unblinded colonoscopy) in the included studies.

Regarding sensitivity for adenomas, the four studies used slightly different outcome measures; nonetheless, heterogeneity between studies was low $\left(\mathrm{I}^{2}=0.0 \%\right)$. Regge et al[28] reported per-patient sensitivity for advanced adenomas or cancer measuring $\geq 6 \mathrm{~mm}$, with CTC detecting 96 of 111 such patients $(86.5 \%)$. No data were presented at a $\geq 10 \mathrm{~mm}$ threshold. Liedenbaum et al[27] reported a $91 \%$ per-patient sensitivity of double-reported CTC for $\geq 6 \mathrm{~mm}$ lesions (of any histology), with 192 of 211 such patients being detected by CTC. Unusually for the CTC literature, this article used a size cut-off before CTC was termed a true-positive: For example, a $4 \mathrm{~mm}$ polyp reported at CTC which was ultimately measured as $6 \mathrm{~mm}$ by the reference standard was regarded as a CTC false-negative, since a CTC finding of a $4 \mathrm{~mm}$ polyp would not typically provoke colonoscopy. In the corresponding report of the same patients[26], a more conventional polyp-matching algorithm was used and results were presented for both double-reported and single-reader CTC. The mean sensitivity of double-reported CTC for $\geq 6 \mathrm{~mm}$ adenomas or carcinoma was $93 \%$ versus $89 \%$ for a single radiologist. Corresponding sensitivities for $\geq 10 \mathrm{~mm}$ adenomas or carcinomas were $95 \%$ and $92 \%$ for double- and single-reporting respectively. Heresbach et al[30] described per-patient sensitivity at $\geq 6 \mathrm{~mm}$ and $\geq 10 \mathrm{~mm}$ thresholds. CTC was $88 \%$ sensitive at the $6 \mathrm{~mm}$ threshold (correctly finding 14 of 16 patients) and $92 \%$ sensitive at the $10 \mathrm{~mm}$ threshold (12 of 13 patients), for both polyps and adenomas. Finally, Sali et al[29] reported per-patient sensitivity for cancer or adenomas measuring $\geq 6 \mathrm{~mm}$, correctly identifying 21 of 22 patients (95.5\%). No per-patient data were presented at a $\geq 10 \mathrm{~mm}$ threshold. These data are summarised in table 3 and the forest plot in figure 2. 
Only one study reported the per-patient sensitivity of colonoscopy for adenomas in comparison to the segmental unblinded reference standard in gFOBt/FIT-positives: Liedenbaum et al[26] found a $98 \%$ sensitivity for adenomas or carcinomas $\geq 6 \mathrm{~mm}$ and a $99 \%$ sensitivity at a $\geq 10 \mathrm{~mm}$ threshold. Heresbach et al[30] reported a per-patient sensitivity for colonoscopy of $99.5 \%$ and $99.7 \%$ for $\geq 6 \mathrm{~mm}$ and $\geq 10 \mathrm{~mm}$ polyps respectively, although did not stratify by gFOBt status. Regge et al[28] found that blinded colonoscopy only missed two advanced adenomas (measuring 13 and $18 \mathrm{~mm}$ ), although whether or not these patients were gFOBt-positive was not stated.

\section{Specificity and positive predictive value}

Overall, specificity varied substantially between studies, ranging from $52 \%$ to $91 \%$ at a $\geq 6 \mathrm{~mm}$ threshold. Consequently, heterogeneity was high $\left(\mathrm{I}^{2}=78.3 \%\right)$, summarised in Table 4 and the forest plot in figure 2 . Since different radiologists in different studies may vary the point at which they judge a test positive, sensitivity and specificity may vary simply because of the arbitrary threshold used by an individual radiologist. Furthermore, there may be differences in the spectrum of cases or sizes of polyps across the studies. A bivariate model was used to construct a summary ROC curve of the included studies (figure 3), taking this into account. None of the moderator covariates (year of publication, number of included participants, prevalence of abnormality, single- or multi-centre design, use of faecal tagging, reader experience or use of three-dimensional interpretation) were found to be significant, perhaps due to the small number of primary studies. From this model, the operating point has average sensitivity of $88.8 \%(95 \% \mathrm{Cl} 83.6$ to $92.5 \%)$ and specificity of $75.4 \%(95 \% \mathrm{Cl}$ 58.6 to $86.8 \%$ ) with the summary curve being reasonably close to the top left corner of ROC space.

\section{Discussion}

CTC is a relatively novel technology that has matured and is now widely available[31]. It is replacing the barium enema for radiological evaluation of the colon, since randomised trials show it is more sensitive and misses fewer cancers and large polyps in older symptomatic adults[12]. The English Bowel Cancer Screening Programme recommends CTC for gFOBtpositive patients who are unsuitable for colonoscopy[8]. However, it is striking how little evidence exists regarding the diagnostic accuracy of CTC in gFOBt/FIT-positive patients. Only four studies have investigated this group, with only two having gFOBT/FIT-positive subjects as their direct focus. National policies are therefore governed largely by extrapolation from these small cohort studies and related reports of higher-risk patient groups.

Nonetheless, the estimated sensitivity of $88.8 \%$ (and range of $86-96 \%$ for the component studies) for adenomas or cancer $\geq 6 \mathrm{~mm}$ suggests that CTC is sufficiently sensitive to substitute for colonoscopy when necessary. Furthermore, heterogeneity was low, implying the (limited) available literature is consistent. This very high sensitivity is greater than that reported in prior meta-analyses of CTC, which range from $69 \%[17]$ to $86 \%[10]$ for $\geq 6 \mathrm{~mm}$ polyps. We suspect this is due to increased average lesion size in our meta-analysis as a consequence of pre-selection by gFOBt/FIT (which preferentially detects larger polyps and cancers via their propensity to bleed). For example, patients with $\geq 1 \mathrm{~cm}$ adenomas/carcinomas heavily outnumbered those with $6-9 \mathrm{~mm}$ neoplasms in our metaanalysis (246 versus 100), whereas this pattern was reversed in a prior, unrestricted metaanalysis[10]. Since CTC is more sensitive for these large lesions, their relative overrepresentation inevitably increases the pooled estimate of CTC sensitivity. Although based on small numbers, pooled sensitivity for cancer was $96 \%(95 \% \mathrm{Cl} 79.8-99.8 \%)$, identical to that derived from a broader meta-analysis of the diagnostic accuracy of CTC[9]. Notably, the sensitivity of colonoscopy for cancer (judged against segmental unblinded colonoscopy) was $95 \%$ in that meta-analysis, implying the two tests have very similar sensitivity for established 
malignancy. Sensitivity for cancer is particularly important since a common reason for performing CTC over colonoscopy is co-morbidity. Detection of smaller adenomas is less crucial, particularly those lacking advanced features. The estimated progression rate of even histologically advanced adenomas to carcinoma is approximately $3-4 \%$ per annum[32], implying that the small chance of missing an advanced adenoma may be acceptable.

Specificity and PPV were less good, with the latter ranging from 62 to $88 \%$, somewhat lower than the $92-93 \%$ reported when CTC is used for asymptomatic screenees[33, 34]. The pooled estimate of specificity was $75.4 \%$, although heterogeneity was high. Low specificities may partly reflect the high prevalence of abnormality in the gFOBt/FIT positive population, potentially leading radiologists to report equivocal findings as positive (to maximise sensitivity). Furthermore, the minimum level of radiologist experience (50 to 100 cases) was substantially lower than the studies reporting high PPV (minimum 300 cases)[33, 34].

Additionally, faecal tagging was not used in the study with the lowest specificity[29], which reported that most of the false-positives were due to faecal residue. Conversely, Regge et al[28] found that most false-positives were due to hyperplastic or diminutive polyps. Irrespective, the implication is that CTC may direct a substantial proportion of normal patients to colonoscopy.

Since the randomised trials supporting gFOBt population screening employed colonoscopy to investigate a positive faecal test result, large-scale screening programmes follow a similar model. CTC is commonly used when colonoscopy is incomplete or contraindicated (including screenee refusal). Patients included in our meta-analysis were, by definition, able to undergo both CTC and colonoscopy. These data are therefore most applicable to a patient population deemed fit for colonoscopy i.e. those with an incomplete colonoscopy or who refuse it for reasons unrelated to their general health. Conversely, the sensitivity of CTC in frailer individuals with relative contraindications to colonoscopy is unknown. Observational data show that cancer and adenoma detection rates by CTC in gFOBt-positives are substantially lower than corresponding detection rates by colonoscopy[35]. However, these screenees were imaged with CTC because colonoscopy was judged inappropriate, meaning that this difference may arise from selection bias rather than reduced sensitivity of CTC. Nonetheless, the high sensitivity and moderate specificity of CTC found in our systematic review may not generalise to frailer patients.

Our review focused on sensitivity and specificity, and did not consider other factors such as safety, patient acceptability or cost. Furthermore, the impact on overall screening compliance by introducing an additional step in the diagnostic pathway (i.e. faecal testing, then CTC, then colonoscopy) is unknown. The high prevalence of abnormality after positive gFOBt/FIT suggested to the authors of one component study[27] that universal adoption of CTC as a "triage test" would not be cost-effective. Conversely, a recent cost analysis concluded that savings would arise via avoiding unnecessary colonoscopy by CTC triage[36].

The major limitation of this study is the small number of studies available in the primary literature for review and meta-analysis. Whilst this is unavoidable, it does imply that our estimates of heterogeneity may be inaccurate, and that the summary estimates may be substantially affected by a single outlying study. It is therefore reassuring that the two largest studies we included[26-28] had almost identical sensitivity and specificity. Additionally, such a small number of component studies precludes meaningful assessment of publication bias via funnel plots or alternatives, meaning that the result of the meta-analysis should be treated with appropriate caution. Assessment of moderator covariates in the bivariate model is also potentially limited by the small number of studies, meaning we may have erroneously discounted these factors as affecting sensitivity or specificity.

In summary, by systematic review we conclude that few studies have directly addressed investigation of $\mathrm{gFOBt} / \mathrm{FIT}$-positive populations by CTC. Nonetheless, available studies 
suggest that the sensitivity of CTC for $\geq 6 \mathrm{~mm}$ adenomas or cancer following a positive $\mathrm{gFOBt} / \mathrm{FIT}$ result is $88.8 \%(95 \% \mathrm{Cl} 83.6-92.5 \%)$. Specificity is more variable between studies and the summary estimate is lower, at $75.4 \%(95 \% \mathrm{Cl} 58.6-86.8 \%)$. Our review suggests that CTC may adequately substitute for colonoscopy when the latter is undesirable or incomplete. The high rate of subsequent testing (predicated by high prevalence of abnormality) and relatively reduced sensitivity of CTC compared to colonoscopy suggests the latter should remain the preferred test where feasible.

\section{Acknowledgements}

See disclosure paragraph 


\section{References}

1. Ferlay J, Shin H-R, Bray F, et al. (2010) Estimates of worldwide burden of cancer in 2008: GLOBOCAN 2008. Int J Cancer J Int Cancer 127:2893-2917. doi: $10.1002 / \mathrm{ijc} .25516$

2. Benson VS, Atkin WS, Green J, et al. (2012) Toward standardizing and reporting colorectal cancer screening indicators on an international level: The International Colorectal Cancer Screening Network. Int J Cancer J Int Cancer 130:2961-2973. doi: 10.1002/ijc.26310

3. Hewitson P, Glasziou P, Irwig L, et al. (2007) Screening for colorectal cancer using the faecal occult blood test, Hemoccult. Cochrane Database Syst Rev CD001216. doi: 10.1002/14651858.CD001216.pub2

4. Benson VS, Patnick J, Davies AK, et al. (2008) Colorectal cancer screening: a comparison of 35 initiatives in 17 countries. Int $\mathrm{J}$ Cancer $\mathrm{J}$ Int Cancer 122:1357-1367. doi: 10.1002/ijc.23273

5. Weller D, Coleman D, Robertson R, et al. (2007) The UK colorectal cancer screening pilot: results of the second round of screening in England. $\mathrm{Br} \mathrm{J}$ Cancer 97:1601-1605. doi: 10.1038/sj.bjc.6604089

6. Tappenden P, Chilcott J, Eggington S, et al. (2007) Option appraisal of populationbased colorectal cancer screening programmes in England. Gut 56:677-684. doi: 10.1136/gut.2006.095109

7. Lee TJW, Rutter MD, Blanks RG, et al. (2012) Colonoscopy quality measures: experience from the NHS Bowel Cancer Screening Programme. Gut 61:1050-1057. doi: 10.1136/gutjnl-2011-300651

8. Taylor SA, Burling DN, Patnick J (2012) Guidelines for the use of imaging in the NHS Bowel Cancer Screening Programme: Second Edition.

9. Pickhardt PJ, Hassan C, Halligan S, Marmo R (2011) Colorectal cancer: CT colonography and colonoscopy for detection--systematic review and metaanalysis. Radiology 259:393-405. doi: 10.1148/radiol.11101887

10. Halligan S, Altman DG, Taylor SA, et al. (2005) CT colonography in the detection of colorectal polyps and cancer: systematic review, meta-analysis, and proposed minimum data set for study level reporting. Radiology 237:893-904. doi: 10.1148/radiol.2373050176

11. De Haan MC, van Gelder RE, Graser A, et al. (2011) Diagnostic value of CTcolonography as compared to colonoscopy in an asymptomatic screening population: a meta-analysis. Eur Radiol 21:1747-1763. doi: 10.1007/s00330011-2104-8

12. Halligan S, Wooldrage K, Dadswell E, et al. (2013) Computed tomographic colonography versus barium enema for diagnosis of colorectal cancer or large polyps in symptomatic patients (SIGGAR): a multicentre randomised trial. Lancet 381:1185-1193. doi: 10.1016/S0140-6736(12)62124-2

13. Atkin W, Dadswell E, Wooldrage K, et al. (2013) Computed tomographic colonography versus colonoscopy for investigation of patients with symptoms suggestive of colorectal cancer (SIGGAR): a multicentre randomised trial. Lancet 381:1194-1202. doi: 10.1016/S0140-6736(12)62186-2

14. Morris EJA, Whitehouse LE, Farrell T, et al. (2012) A retrospective observational study examining the characteristics and outcomes of tumours diagnosed within and without of the English NHS Bowel Cancer Screening Programme. Br J Cancer 107:757-764. doi: 10.1038/bjc.2012.331

15. Bretagne J-F, Manfredi S, Piette C, et al. (2010) Yield of high-grade dysplasia based on polyp size detected at colonoscopy: a series of 2295 examinations following a positive fecal occult blood test in a population-based study. Dis Colon Rectum 53:339-345. doi: 10.1007/DCR.0b013e3181c37f9c 
16. Kolligs FT, Crispin A, Graser A, et al. (2013) Risk factors for advanced neoplasia within subcentimetric polyps: implications for diagnostic imaging. Gut 62:863870. doi: $10.1136 /$ gutjnl-2011-300111

17. Chaparro M, Gisbert JP, Del Campo L, et al. (2009) Accuracy of computed tomographic colonography for the detection of polyps and colorectal tumors: a systematic review and meta-analysis. Digestion 80:1-17. doi: $10.1159 / 000215387$

18. Hassan C, Pickhardt PJ, Kim DH, et al. (2010) Systematic review: distribution of advanced neoplasia according to polyp size at screening colonoscopy. Aliment Pharmacol Ther 31:210-217. doi: 10.1111/j.1365-2036.2009.04160.x

19. Taylor SA, Laghi A, Lefere P, et al. (2007) European Society of Gastrointestinal and Abdominal Radiology (ESGAR): consensus statement on CT colonography. Eur Radiol 17:575-579. doi: 10.1007/s00330-006-0407-y

20. Barish M (2003) Consensus statement. Proc. 4th Int. Symp. Virtual Colonoscopy. Boston, Mass, pp 137-143

21. Whiting PF, Rutjes AWS, Westwood ME, et al. (2011) QUADAS-2: a revised tool for the quality assessment of diagnostic accuracy studies. Ann Intern Med 155:529-536. doi: 10.7326/0003-4819-155-8-201110180-00009

22. Viechtbauer W (2010) metafor: Meta-Analysis Package for R. J Stat Softw 36:148.

23. $R$ Core Team $R$ : A language and enviroment for statistical computing. $R$ Foundation for Statistical Computing, Vienna.

24. Gatsonis C, Paliwal P (2006) Meta-analysis of diagnostic and screening test accuracy evaluations: methodologic primer. AJR Am J Roentgenol 187:271281. doi: 10.2214/AJR.06.0226

25. Reitsma JB, Glas AS, Rutjes AWS, et al. (2005) Bivariate analysis of sensitivity and specificity produces informative summary measures in diagnostic reviews. J Clin Epidemiol 58:982-990. doi: 10.1016/j.jclinepi.2005.02.022

26. Liedenbaum MH, de Vries AH, van Rijn AF, et al. (2010) CT colonography with limited bowel preparation for the detection of colorectal neoplasia in an FOBT positive screening population. Abdom Imaging 35:661-668. doi: 10.1007/s00261-009-9586-8

27. Liedenbaum MH, van Rijn AF, de Vries AH, et al. (2009) Using CT colonography as a triage technique after a positive faecal occult blood test in colorectal cancer screening. Gut 58:1242-1249. doi: 10.1136/gut.2009.176867

28. Regge D, Laudi C, Galatola G, et al. (2009) Diagnostic accuracy of computed tomographic colonography for the detection of advanced neoplasia in individuals at increased risk of colorectal cancer. JAMA J Am Med Assoc 301:2453-2461. doi: 10.1001/jama.2009.832

29. Sali L, Falchini M, Monica P, et al. (2010) CT colonography before colonoscopy in subjects with positive faecal occult blood test. Preliminary experience. Radiol Med (Torino) 115:1267-1278. doi: 10.1007/s11547-010-0569-1

30. Heresbach D, Djabbari M, Riou F, et al. (2011) Accuracy of computed tomographic colonography in a nationwide multicentre trial, and its relation to radiologist expertise. Gut 60:658-665. doi: 10.1136/gut.2010.225623

31. Boone D, Halligan S, Taylor SA (2011) Evidence review and status update on computed tomography colonography. Curr Gastroenterol Rep 13:486-494. doi: 10.1007/s11894-011-0217-5

32. Brenner H, Hoffmeister M, Stegmaier C, et al. (2007) Risk of progression of advanced adenomas to colorectal cancer by age and sex: estimates based on 840,149 screening colonoscopies. Gut 56:1585-1589. doi: 10.1136/gut.2007.122739

33. Pickhardt PJ, Wise SM, Kim DH (2010) Positive predictive value for polyps detected at screening CT colonography. Eur Radiol 20:1651-1656. doi: $10.1007 / \mathrm{s} 00330-009-1704-z$ 
34. Zueco Zueco C, Sobrido Sampedro C, Corroto JD, et al. (2012) CT colonography without cathartic preparation: positive predictive value and patient experience in clinical practice. Eur Radiol 22:1195-1204. doi: 10.1007/s00330-011-23670

35. Plumb AA, Halligan S, Nickerson C, et al. (2013) Use of CT colonography in the English Bowel Cancer Screening Programme. Gut. doi: 10.1136/gutjnl-2013304697

36. Sweet A, Lee D, Gairy K, et al. (2011) The impact of CT colonography for colorectal cancer screening on the UK NHS: costs, healthcare resources and health outcomes. Appl Health Econ Health Policy 9:51-64. doi:

$10.2165 / 11588110-000000000-00000$ 


\section{Table legends}

\section{Table 1:}

Characteristics of the included studies. $\mathrm{RI}=$ reconstruction interval, $\mathrm{CAD}=$ computerassisted detection. ${ }^{*}$ Regge et al reported results for histologically advanced adenomas.

\section{Table 2:}

QUADAS-2 quality assessment of the included studies

\section{Table 3:}

Per-patient sensitivity (95\% confidence intervals) and negative predictive value (95\% $\mathrm{CI}$ ) of CTC. $\mathrm{Sn}=$ sensitivity, NR=not reported, NPV=negative predictive value. Data from the Liedenbaum et al studies is for single-reader CTC.

\section{Table 4:}

Per-patient specificity (95\% confidence intervals) and PPV $(95 \% \mathrm{Cl})$ of CTC. $\mathrm{Sp}=$ specificity, NR=not reported, $\mathrm{PPV}=$ positive predictive value. Data for the Liedenbaum et al studies is for single-reader CTC. 


\section{Figure legends}

\section{Figure 1}

Flowchart of the systematic review.

\section{Figure 2}

Forest plot of included studies showing individual and pooled estimates of sensitivity and specificity of CTC for $\geq 6 \mathrm{~mm}$ adenomas and cancers (Regge et al reported histologically advanced neoplasia). For each study, marker area is proportional to precision, with greater precision indicated by larger area. Pooled values are derived from the bivariate random effects model. $\mathrm{TP}=$ true positive, $\mathrm{FN}=$ false negative, $\mathrm{TN}$ $=$ true negative, $\mathrm{FP}=$ false positive.

\section{Figure 3}

Summary ROC curve of included studies. The sensitivity of each individual study for $6 \mathrm{~mm}$ adenomas or cancer is plotted against 1-specificity. Regge et al (square) reported advanced adenomas only. Data for Liedenbaum et al (circle) are for single reader CTC. Heresbach et al and Sali et al are represented by a triangle and diamond respectively. Grey lines show 95\% confidence regions of each individual study. Black circle shows the overall estimate at the operating point. 


\section{Appendix}

Studies excluded from the systematic review after full-text reviewed

\begin{tabular}{|l|c|l|}
\hline Author & Year & Reason for exclusion \\
\hline Fenlon & 1999 & FOBt-positive patients not reported separately \\
\hline Pescatore & 2000 & FOBt-positive patients not reported separately \\
\hline Yee & 2000 & FOBt-positive patients not reported separately \\
\hline Laghi & 2002 & FOBt-positive patients not reported separately \\
\hline Laghi & 2002 & FOBt-positive patients not reported separately \\
\hline Macari & 2002 & FOBt-positive patients not reported separately \\
\hline Wong & 2002 & FOBt-positive patients not reported separately \\
\hline lannaconne & 2003 & FOBt-positive patients not reported separately \\
\hline lannaconne & 2003 & FOBt-positive patients not reported separately \\
\hline Pineau & 2003 & FOBt-positive patients not reported separately \\
\hline Yee & 2003 & FOBt-positive patients not reported separately \\
\hline Cohnon & 2004 & FOBt-positive patients not reported separately \\
\hline Cotton & 2004 & FOBt-positive patients not reported separately \\
\hline Hoppe & 2004 & FOBt-positive patients not reported separately \\
\hline lannaconne & 2004 & FOBt-positive patients not reported separately \\
\hline lannaconne & 2005 & FOBt-positive patients not reported separately \\
\hline Rockey & 2005 & FOBt-positive patients not reported separately \\
\hline Haykir & 2006 & FOBt-positive patients not reported separately \\
\hline Reuterskiold & 2006 & FOBt-positive patients not reported separately \\
\hline Selcuk & 2006 & FOBt-positive patients not reported separately \\
\hline Carrascosa & 2007 & FOBt-positive patients not reported separately \\
\hline Walleser & 2007 & FOBt-positive patients not reported separately \\
\hline Roberts-Thomson & 2008 & FOBt-positive patients not reported separately \\
\hline Sali & 2008 & Only some patients underwent the reference test \\
\hline Fisichella & 2009 & FOBt-positive patients not reported separately \\
\hline Nagata & 2009 & FOBt-positive patients not reported separately \\
\hline Neri & 2009 & Only some patients underwent the reference test \\
\hline Ozsunar & 2009 & FOBt-positive patients not reported separately \\
\hline Sali & 2013 & Only some patients underwent the reference test \\
\hline
\end{tabular}

